\title{
Co-evolutionary analysis of the immune and nervous systems may reveal novel diagnosis and treatment options for mental health and neurodegenerative disorders
}

Catarina Cunha ${ }^{1 *}$

Emotional Brain Institute, Nathan Kline Institute, Orangeburg, NY, USA

${ }^{*}$ Correspondence: Catarina Cunha, Emotional Brain Institute, Nathan Kline Institute, Orangeburg, NY, USA,

E-mail: catarina.cunha@nki.rfmh.org

\section{Abstract}

Theories regarding the co-evolution of the immune system and neuronal network have been discussed throughout science history, but only recently research has shown evidence of the immune system modulating the neuronal circuitry of social behavior directly. Targeting the communication of the two systems would be an important novel approach that holds the potential for discoveries and development of precise diagnosis and treatments, and possibly even prevention of illnesses such as Parkinson, Alzheimer, and autism. We hypothesize, that there are 3 ways for the system to be dysregulated in pathologies: 1) the neuronal network is mal-functioning by default and derails the immune system, 2) basically vice versa and both, 3) the immune system gets triggered too early too much or chronically (inflammation, pollution, stress, etc), which then leads to aberrations of the central nervous system and its behavioral responses, however, even in this scenario some organisms cope with chronic stressors, displaying resilience, and others don't. We hypothesize, that downregulation or blockage of immune-neuronal network communication would be an important target for treatment or even prevention of various illnesses, including neurological and mental health disorders. We will discuss various mechanisms supporting this co-evolution hypothesis, including cytokines modulating behavior and neurotransmitters modifying immune responses, and novel targets that could be potentially utilized for novel treatment development. In the light of fast increasing rate of mental health and neurological illnesses, we need to establish the risk level in individuals for aberrant immune-neuronal system/communication-related disorders development, which will play an important role in sustaining stable public health and therefore also a steady economy. In the future, the development of novel screening and analyzing 
technology will lead to cost-effectiveness, enabling us to utilize these individualized preventive screenings as broadly applied tools.

\section{Introduction}

The first multicellular organisms were the Ediacaran biota, which were tubular and frond-shaped and mostly sessile organisms; these organisms existed without predators and flourished for ca. 300 million years until the beginning of the Cambrian Period [1]. Researchers were unable to find fossils, of the Ediacaran period, with sensory input systems such as light receptors. As far as we know, the organisms which existed at that time did not interact with one another and there is no evidence of engagement with their environment; this means that the first information transmission (or "nervous") system was for controlling internal coordination and not sensory-motor control [2]. However, even in "The Garden of Ediacara" bacteria and viruses existed, which means, that the threat of infections was always present, representing a form of input information. In order to process and react to that input information, we theorize that an early form of the immune system was developed, which would mean, that the immune system was the first input information processing mechanism, which then influenced the internal information system (neuronal network) and the organism's "behavior". Furthermore, the crucial role of the immune system consists of the discrimination between self- versus non-altered-self which enables the system to enforce host defense against invading microbes and also tumors. The co-evolution of the immune system and the neuronal network has been proposed before [3,4], and recent research has shown a direct link of the immune system influencing the neuronal circuitry of social behavior [5]. Extrapolating this hypothesis to disorders could lead to novel discoveries and development of effective diagnosis and treatments, and maybe even prevention of illnesses such as Parkinson, Alzheimer, and autism. There are 3 ways how the whole system could be going wrong, and there is accumulating evidence for all options: 1) the neuronal network is mal-functioning by default and influences the immune system, 2) basically vice versa and both, 3) the immune system gets triggered too early too much or chronically (infections, pollution, stress, etc), which then leads to aberrations of the central nervous system and its behavioral responses, although even 
in this scenario there are organisms that cope with the chronic stressors (resilience), and others don't [5-12].

\section{Evidence supporting the hypothesis:}

\section{Cell organization}

Cytoskeletal organization and signaling functions, have been shown in both systems to involve a common scaffolding protein, the microtubule-organizing center protein RanBPM, and its multiple functional domains seem to have pleiotropic functions within both systems, consisting of neurite branching [13], association with adhesion molecules [14], and signaling in the cytoplasm of lymphoid cells [15].

\section{Memory}

Both systems use a "memory", meaning that cells "remember" what they had experienced in the past and are able to recall these experiences given a specific stimulus which proposes common underlying mechanisms.

\section{Information transfer/communication}

There is evidence that both systems share common communication mechanisms, which include cytokines, chemokines, neuropeptides, neurotransmitters, neurotrophins [16], and their receptors [17]. It has been recently shown, that chemokines and their receptors, such as CXCL12-CXCR4, are not only used by lymphoid cells, in cell trafficking and immune responses, but that they also play an important role in neuronal cell migration, development, and cell-cell communication within the nervous and immune systems [18].

Other examples are Toll-like receptors (TLRs), that were first described in immune cells, however, they were shown to be also present in neuronal cells performing a cell-autonomous function [19]. During injury, autoimmune responses, hypoxia, and neurodegeneration TLR8 was recorded to activate cells in the CNS through ligands [20], which leads to suppression of 
neurite outgrowth and induction of apoptosis. TLR3 seems to be activated during viral infections, which is proposed to elicit an "immune"-type response in neurons characterized by cytokine production [21].

The cytokine interleukin-6 (IL-6) was first characterized as a B-cell differentiation factor, which is involved in mechanisms of maturation of $B$ cells into antibody-producing cells. Later, it was shown that IL-6 also plays roles in other physiological systems, which includes the nervous system. IL-6 is involved in neurogenesis (neurons and glial cells) in mechanisms of mature neurons and glial cells in normal conditions and in injury/stress models. The cytokine family IL-6 has been labeled as neuropoietins, for the reason that they behave in a neurotrophin-like way. The expression mechanisms of IL-6 were detected to be affected in various animal models of neuropathology and suggest being a target for treatments [22]. In vitro, IL-1 $\beta$ acts as a stimulator of IL-6 production [23, 24]. Mechanical injury was shown to upregulate IL-6 in adult human astrocytes [25]. The expression of IL- 6 by astrocytes is under control of the autocrine regulation by which IL-6, and the addition of sIL-6R cooperates with IL-1 $\beta$ and TNFa, which incites IL-6. Oncostatin M (OSM) was shown to be able to generate IL-6 production on its own, but in combination with TNFa, it leads to higher IL-6 expression [26]. Also, IL-17 has been recorded to react with IL-6 (+ sIL-6R) to elicit IL-6 production in astrocytes [27].

IL-12 was demonstrated to play a role in inducing or propagating inflammatory skin lesions. Studies detected the presence of IL-12 in neural tissue, which proposes that the nervous system is modifying and/or amplify implicated immune responses [28].

IL-1 activity is stimulated by fever and has been shown to modulate peripheral sensory neurons activity. Additionally, new studies recorded that sensory afferent neurons express receptors for TNF, IL-1, LPS, and other products of inflammation. The immune system uses these mechanisms to elicit neural reflex circuits underlying behavioral and physiological responses involved in acute and chronic immune mechanisms such as acute phase protein responses, fever, anorexia, insulin resistance, chronic sickness behavior, depression, and cachexia [29, 30]. These mechanisms prevent non-resolving inflammation, in which pro-inflammatory phagocytes switch to an anti-inflammatory phenotype that promotes tissue 
repair rather than tissue destruction [31]. Especially TGF- and IL-4 were shown to induce macrophages to express an alternative anti-inflammatory phenotype. This evidence suggests, that the immune system uses its mediators to enforce inflammation suppression through nervous system activation, and vice versa [30, 32].

Prostaglandins are lipid messenger molecules, which have a variety of functions including participation in regulating inflammation and pain. Phospholipase A2 induces arachidonic acid release from phospholipids, that are then converted by cyclooxygenase enzymes to prostaglandin $\mathrm{H} 2$, which represents the precursor of various prostaglandins.

Studies have shown effects of prostaglandins on ion channels in neurons, wherein especially PGE2 and prostacyclin PGI2 have diverse effects, seeming to generally sensitize nerve cells to electrical or chemical stimuli both at the peripheral terminal of nociceptors at the site of tissue injury and at the synapses in the spinal cord. The mechanism responsible for the mostly facilitatory prostaglandin effects, function preferentially by their binding on to EP and IP G-protein coupled membrane receptors, which activate enzymes such as adenylate cyclase and phospholipase C (PLC) leading to an increase of cAMP and consequential activation of protein kinase $A(P K A)$ or PKA-independent effects of CAMP, which is mediated by Epac (exchange protein activated by cAMP). The activation of PLC induces an increase of inositol phosphates and cytosolic calcium. These mechanisms are known to be involved in synaptic plasticity and long term memory and promise to be a novel target for the development of new analgesics [33]. Recent research explored specific regulation of EPAC isoforms (EPAC1 and EPAC2) for CAMP-based therapies, where EPAC2-selective agonists were proposed to promote insulin secretion from pancreatic $\beta$ cells, whereas EPAC1-selective agonists may be useful in the treatment of vascular inflammation [34]. We propose, that the design of EPAC-selective agonists or antagonists may also present a promising pathway for novel strategies in mental health, neurodegenerative disorders, and neuropathy.

The other way around, neuropeptide Y (NPY), was assumed to be expressed exclusively in the central and peripheral nervous systems. However, a new study showed its occurrence in lymphocytes, which once being triggered by its receptor $\mathrm{Y} 1$ generates pleiotropic effects on 
cell migration, cytokine release, and antibody production, while being involved in autoimmunity and inflammation processes [35]. Also, endocannabinoids have been shown to be involved in both, immune responses and adult neurogenesis [36].

\section{Direct communication of the two systems}

Some immunologists adopted the term "synapse", which was traditionally used in the nervous system, describing a formed shared communication surface platform for interaction of immune and neuronal cells. The existence of functional synapses between neurons and lymphoid cells have been hypothesized before. Supporting this evidence, a study showed that agrin, a matrix molecule, plays a role in the structure of synapses in both systems [37].

Functionally, contacts between the two cell types have been described in pathophysiological situations, such as autoimmune disorders, where lymphoid cells attack neurons. Physiological heterologous connections have also been described in lymphoid organs, where sympathetic innervation and production of neuropeptides or norepinephrine affect the functioning of lymphoid cells, however, the exact underlying mechanisms are still unclear. To summarize, synaptic communication, as well as cytokines and neuromodulatory substances, were found to form an intricate network of communication pathways that affect both systems and their cell types [38].

IFN-y represent a co-evolutionary link between behavior and an anti-pathogen immune response

Recently, there have been studies showing that meningeal immunity influences spatial learning and memory, and even social behavior. Examination of underlying mechanisms uncovered that inhibitory neuron activity is directly modulated by IFN- $y$. Additionally, an increase of GABAergic ( $\gamma$-aminobutyric-acid) currents in projection neurons by induced by IFN- $\gamma$ was recorded, showing a direct molecular link between meningeal immunity and neural circuits involved in social behavior. Meta-analysis of the transcriptomes of a range of organisms reveals that rodents, fish, and flies elevate IFN-Y/JAK-STAT-dependent gene signatures in a 
social context, supporting the hypothesis of a co-evolutionary link between behavior and an anti-pathogen immune response [5].

\section{The synergy of both systems}

Group 2 innate lymphoid cells (ILC2s), were shown to be entangled with cholinergic neurons. Furthermore, ILC2 cells express a receptor for neuromedin $U$ (NMU), which was first described as a neurotransmitter. Exposure of ILC2 cells to NMU-induced their multiplication, and secretion of cytokines triggering immune responses and/or causing inflammation. An example of this system being involved in disorders was shown in the lungs affected by allergic lung inflammation where NMU amplifies ILC2-driven responses [39].

\section{Migration and development}

Another common characteristic of both systems is plasticity in developmental fates, in which, depending on environmental cues, cells are guided into one of the multiple differentiation options [40, 41]. An example for the neural system is represented by neural crest cells, of which multi lineage progenitors can differentiate into a variety of cell types such as neurons, glia, perivascular cells, bone, cartilage, or smooth muscle $[42,43]$. In the immune system $T$ cells develop a variety of functional characteristics, differentiating into cells with specific tasks within immune responses (Th1, Th2, Th17 cell, etc.) [44]. Rarely plasticity is observed in cells, that are already committed to one cell type, which is later reversed by acquiring a different functional identity $[45,46]$.

$\mathrm{RET}$, a tyrosine kinase receptor, has been identified to play a crucial role in the development of the gut nervous system, which was also found to be important for lymphoid organogenesis within the same organ [47], proposing that multicellular, multiorgan life forms seem to utilize similar mechanistic tools to arrange the development and organization of different tissues within one organ. RET is involved in mechanisms underlying the migration of neural crest cells in the gut wall and their differentiation and aggregation to form the ganglia of the enteric neural plexus. Additionally, RET plays a role in the development of Peyer's patch primordia, which is formed by the aggregation of hemopoietic cells colonizing the gut wall during embryogenesis. 
Data shows, that RET ligand GDNF and its cognate co-receptor GFRa1 participate in the formation of its enteric nervous system, however, is not involved in the formation of Peyer's patch primordia. This suggests, that RET may play a role in both systems proposed by its differential temporal and spatial availability of its ligands and co-receptors [48].

Semaphorins are signaling molecules that play a crucial role in neuronal guidance and their processes' extensions [49]. Recently, they were found to be also expressed by lymphocytes for their movement in a precise fashion in the same organ [50].

Furthermore, the NF-kB and Ikaros families are transcription factors, which were shown to play a significant role in the development and function of cells in both, the neuronal and immune system [51]. Recently, the aryl hydrocarbon receptor (AHR), which is a member of the basic helix loop helix (bHLH) family, was shown to respond to endogenous ligands involved in normal physiology and development of both systems [52].

The specialized gene regulation mechanism of "allelic exclusion", was detected to be utilized in both systems allowing for the expression of only one gene out of a large array of similar genes [53]. Examples of genes utilizing this mechanism are olfactory receptor genes in the nervous system and antigen-specific receptor genes in B and T cells of the immune system [53].

\section{Vagus nerve}

Later it has been established, that adrenal hormones also hold anti-inflammatory qualities, including ameliorating effects against shock and rheumatoid arthritis. Research done in the 1960s showed that electrical stimulation of the vagus nerve increased the release of acetylcholine from the spleen [98]. Anatomy studies demonstrated the innervation from nuclei residing in the brain stem, the sympathetic chain, and peripheral ganglion cells, of principal organs of the immune system, through nerve endings on within nanometers of immune cells that express receptors for acetylcholine, catecholamines, neuropeptides, and other neurotransmitters. Linda Watkins discovered in the 1990s, that neuroscience and immunology directly intersected by showing that a fever response in rodents, elicited by intra-abdominally 
administration of IL-1, required an intact vagus nerve [29, 30], meaning that action potentials transmitted in sensory nerve fibers to the brainstem transmit the signal of inflammation in peripheral tissues. Niijima demonstrated that action potentials travel in the vagus nerve from the liver to the brainstem, which activates descend neurotransmission to the spleen, thymus, and other organs [99]. Additionally, it was demonstrated, that an intact vagus nerve is crucial for CNI1493 to inhibit cytokine release in vivo, proposing that afferent and efferent signals transmitted in the vagus nerve are components of an inflammatory reflex, which presents $s$ mechanisms of a neural circuit modulating innate immune responses [100]. These facts suggest that modulation of the vagus nerve activity would be a treatment option.

\section{Neuromodulators}

The noradrenergic (NE) system is a rapid-acting neuromodulator implicated in coordinating attentional, emotional and mnemonic mechanisms [69, 70], and synaptic strength [71-75]. Chronic hyperresponsiveness of the HPA axis and NE system and corticotrophin-releasing hormone $(\mathrm{CRH})$ system is associated with mental health disorders and cardiovascular problems [76]. However, targeting NE receptors in the amygdala can counteract aversive memories in animals and humans [77-79]. It also has been shown that maternal deprivation may lead to excessive stress responses in adulthood, which is accompanied by higher noradrenergic release. Underlying mechanisms were proposed to be driven by epigenetics, where signaling cascades downstream of NE receptors comprise protein kinase (ERK) and rapamycin (TOR), which regulate various gene expression mechanisms [59, 80-85], and formation of long-term potentiation (LTP) and de-potentiation (LTD), representing the synaptic model for memory [86-89]. Enforcement of LTP through NE was shown to require DNA methylation and also histone acetylation, however, inhibition of histone deacetylases (HDACs) did not contribute to further enhancement of NE-induced LTP [90].

Non Resolution of inflammation increases the pathogenesis of sepsis, atherosclerosis, obesity, cancer, pulmonary disease, inflammatory bowel disease, neurodegeneration, multiple sclerosis, and rheumatoid arthritis [91]. Aberrations in glucocorticoids and/or pituitary hormones regulating their production induce sensitivity to inflammation and infection [92]. The 
onset of inflammation or stress increases glucocorticoid levels, which induce feedback signals to suppress an immune response, that provides a protective mechanism from toxicity and tissue damage by excessive inflammation. Macrophage-derived TGF was demonstrated to be an anti-inflammatory mediator deactivating macrophages; IL-10 was also shown to be an inflammation-resolving humoral mediator produced by monocytes [93]; more anti-inflammatory mediators consist of soluble cytokine receptors, cytokine receptor antagonists, eicosanoids, and oxygenated and nitrated lipids [91]. The afferent neurons express receptors for TNF, IL-1, LPS, and more signal transmitters of inflammation, which activate neural reflex circuits underlying behavioral and physiological responses associated with acute and chronic immune responses, such as acute phase protein responses, fever, anorexia, insulin resistance, chronic sickness behavior, depression, and cachexia [29, 30].

Efferent of the reflex circuit consists of neuronal signals that travel to the organs using acetylcholine and norepinephrine as neurotransmitters. Administration of propranolol to inhibit adrenergic signaling decreased the risk of bacterial infection significantly, however, adrenergic-mediated aberrations in lymphocyte activity was shown to increase the risk of bacterial infection, in which adrenergic neurons projecting to the liver regulate hepatic invariant natural killer T (iNKT) cells promoting systemic immunosuppression [94]. These results present an important target to treat lesions to the CNS associated with immunosuppression, including head trauma, Alzheimer's and other neurodegenerative diseases, and sepsis survivors. Especially, because until this day, there is no specific test for ALS, its assessment remains a clinical diagnosis, and the usefulness of these test schemes is still unclear [95]. We propose that aberrant acitivity of transposons (TE) presenting a viral origin of immune mechanisms drive the malfuntion in immune responses leading to the observed neuronal damage [96, 97]. Screening for TE activity in patients would be an important step towards development of precise clinical diagnosis.

\section{Serotonin}

Serotonin (5-hydroxytryptamine; 5-HT) was first described as a monoamine neurotransmitter. However, numerous studies showed, that its biological function is versatile influencing, reward, 
learning, memory, synaptic plasticity, cognition, and various physiological processes. 5-HT plays an important role in stress responses, and its aberrations have been shown to be involved in different mental health disorders, which lead to drug developments targeting the 5-HT system for mental illnesses. 5-HT affects in a concentration-dependent manner metabolism in various cell types, such as neurons, enterochromaffin cells, adipocytes, pancreatic beta-cells, fibroblasts, smooth muscle cells, epithelial cells, and leukocytes. In all of these cells, three different types of genes are continuously being expressed and/or activated, which regulate 5-HT function, consisting of the membrane proteins (response to 5-HT) SERT, 5HTR-GPCR, and 5HT3-ion channels; downstream signaling transduction proteins; and the enzymes regulating 5-HT metabolism IDO and MAO, that activate catabolites, such as melatonin, kynurenines, and kynurenamines. Therefore, 5-HT is involved in various immunological mechanisms, including chemotaxis, leukocyte activation, proliferation, cytokine secretion, energy, and apoptosis. These systems play a role in illnesses which involve both, an immune system and neuronal system component, such as major depression, fibromyalgia, Parkinson's, Alzheimer disease, psoriasis, arthritis, allergies, and asthma [7].

The enzyme limiting the levels of 5-HT synthesis is tryptophan hydroxylase (TPH), of which the TPH isoform (TPH2) has been shown to be neuron-specific, and whose gene expression is modulated by various internal and external environmental factors, such as the biological clock, stressors, endogenous hormones, and is of both pathophysiological and pharmacological significance [54].

A range of serotonergic system candidate genes have been investigated for association with mental health disorders, such as suicidal behavior. Studies examined the involvements of serotonin transporter 5 ' functional promotor variant, serotonin $5-\mathrm{HT} 1 \mathrm{~A}, 5-\mathrm{HT} 1 \mathrm{~B}$, and $5-\mathrm{HT} 2 \mathrm{~A}$ receptors, monoamine oxidase $A$ (an enzyme responsible for the degradation of serotonin), and TPH1, TPH2, but the results were inconclusive [24]. The only candidate seeming to be more convincing is the serotonin transporter 5' promoter variant (5-HTTLPR), showing an association between the low-expressing $S$ allele and suicide [1], however, this connection has not been supported in stress-responsive depression. 
Aberrations in this 5-HT circuit have been proposed to induce hypercortisolemia and the increase in pro-inflammatory cytokines observed in depression. Glucocorticoids and pro-inflammatory cytokines increase the conversion of tryptophan to kynurenine, which on the other hand results in decreased synthesis of serotonin, leading to the formation of neurotoxins comprising the glutamate agonist quinolinic acid, which contributes to the increase in apoptosis of astrocytes, oligodendroglia, and neurons. Anti-inflammatory drugs would provide a promising new treatment option as antidepressants and other mental health/neurological disorders [55].

\section{Breakdown of $5-H T$}

There are four pathways of $5-\mathrm{HT}$ breakdown within the immune system. In one of the pathways, $5-\mathrm{HT}$ is acetylated by arylalkylamine $\mathrm{N}$-Acetyltransferase (AANAT; EC: 2.3.1.87) producing $\mathrm{N}$-acetyl $5-\mathrm{HT}$, acquiring then a methyl group from $\mathrm{N}$-acetylserotonin-O-methyltransferase (ASMT; EC: 2.1.1.4; previously known as hydroxyndole-O-methyltransferase, HIOMT) to generate melatonin [7]. Melatonin is known to play an important role in the circadian rhythm, which influences the sleep-wake cycle, hormone release, eating habits/digestion, body temperature, mood, and behavior. Low tryptophan levels may decrease serotonin and melatonin levels, leading to detrimental effects seen in many mental health disorders [56]. Research showed, that 1,25-Dihydroxyvitamin $D(1,25 D)$ controls the expression of TPH2, in which TPH2 mRNA was induced 2.2-fold by $10 \mathrm{nM}$ 1,25D in human U87 glioblastoma cells and 47.8-fold in rat serotonergic RN46A-B14 cells [57]. Physiological abnormalities of in melatonin levels have been shown in various mental health disorders including autism (ASD), where lower nighttime melatonin or melatonin metabolite concentrations were reported compared to controls. The mentioned mechanisms may be directly attributed to variations of the melatonin pathway physiology in people affected by ASD [56]. Additionally, this circuit could be an important direct link mechanism between aberrant immune response and mental health disorders such as ASD, in which environmental immune stressors such as pollution has been shown to play an important role. Especially during development organisms seem to be more vulnerable to these stressors, as shown in two 
studies conducted in communities around the US, which showed a specific association with air pollution exposure during the third trimester and ASD [10]. Detecting the risk factor for developing aberrant immune-neuronal system communication during development and targeting the miscommunication may provide an important tool for disorder prevention in the future.

\section{Epigenetics and 5-HT}

As mentioned above, TPH2 is involved in the function of $5-\mathrm{HT}$ and is associated with different psychopathologies, such as major depressive disorder (MDD) and suicidal behavior. Epigenetic mechanisms were shown to modulate gene expression of TPH2 by the methylation of a single $\mathrm{CpG}$ site in its promoter region. A recent study showed that TPH2 expression was regulated by DNA methylation of the TPH2 promoter region in patients with major depression disorder (MDD) and that there was a significant correlation between the methylation levels of the TPH2 promoter and levels of depression, hopelessness and cognitive impairment, and even risk of suicide [58].

Common pathways and epigenetic mechanism in neuronal systems and immune response TOR is a serine-threonine kinase, which is part of the PI3K/Akt/TOR signaling pathway, that plays a role in several cell functions, such as growth, proliferation, apoptosis, and autophagy. Modulation of the TOR pathway via epigenetic mechanisms involving DNA methylation and histone modifications was shown to play a role in memory reconsolidation. It was found that memory retrieval was associated with transient suppression of TOR pathway (activation of autophagy) while the subsequent reconsolidation was linked to TOR pathway activation (suppression of autophagy), both involving epigenetic regulatory changes [59].

The TOR pathway is inhibited by the TSC complex, which is regulated by several inputs, such as the activation of the AMP kinase (AMPK), which detects reduced intracellular ATP levels and the decline of insulin receptor signaling feedback to a drop in systemic growth signals [60]. This circuit regulates a balance between translation and resources/energy level. A recent study investigated the TSC inhibition of TOR upon infection. The results show that elF2a 
phosphorylation through GCN2 activation and 4E-BP hypo phosphorylation through AMPK-TSC inhibition of TOR activity suppress host translation after infection [61].

The stress-activated protein kinase (SAPK) c-Jun N-terminal kinase (JNKs), is another of multifunctional-signaling molecules involved epigenetic mechanisms that play a role in the immune system and neuronal system, which are activated in response to various cellular stressors and in response to inflammatory mediators. JNKs were shown to be involved in brain development, repair, and memory formation, regulation of neuroinflammation and neuronal death, and to be crucial for normal immune and inflammatory response. Deviations of the JNK activity has been involved in the pathogenesis of Alzheimer's disease, and its normal activity plays a role in normal aging. JNK was demonstrated to play a crucial role in regulating pro-inflammatory cytokines biosynthesis at the transcriptional and translational level. The inhibition of JNK has been shown to constrict microglial activity and the release of neurotoxic chemicals including pro-inflammatory cytokines.

Research demonstrated, that endoplasmic reticulum (ER) stress suppresses insulin receptor signaling through hyperactivation of JNK and consequential serine phosphorylation of insulin receptor substrate-1 (IRS-1). These findings demonstrate that the TOR and JNK pathway share common functions and may offer novel opportunities for treatments [60].

Rap1 and 2, JNK, and their regulators are present at excitatory synapses. A study showed in hippocampal CA1 neurons that Rap2-JNK signaling pathway receives its inputs from synaptic and NR2A-containing NMDA-Rs. After that, Rap2-JNK activity mediates synaptic removal of GluR1- and GluR2L-containing AMPA receptor (-R), which is crucial for depotentiation in the neurons. In this system, related small GTPases Ras and Rap1 are also important for signaling synaptic AMPAR trafficking during long-term potentiation (LTP) and long-term depression (LTD), respectively. Rap1-p38 MAPK signaling represents the LTD mechanisms, which is stimulated by NR2B-containing NMDA-R. To summarize, Rap2 activity induces de-potentiation and complements the LTD mechanism driven by Rap1 signaling and opposes Ras signaling (LTP) in the bidirectional control of synaptic strength [62]. The genes JNK-1, JNK-2, and 
JNK-3, encoding for ten different isoforms were shown to be increased in neurodegenerative disorders such as in the brain tissue of AD patients [63].

Interestingly, a study recently showed the connection between JNK and TOR pathway, demonstrating, that MAPK/JNK is a positive regulator of TORC1 signaling and its inactivation provides an inducing signal for the coordinated autophagy and synaptic pruning, which is also affected by immune function [64].

\section{Drugs targeting TOR and JNK}

There are two different forms of TOR, mTORC1, and mTORC2, presenting targets for treatments. Rapamycin is a selective inhibitor of mTORC1, but not in mTORC2. TOR ATP-competitive inhibitors have been reported to act on both TOR complexes. Research showed, that some compounds originally developed as PI3K inhibitors also target TOR because it shares high sequence homology in the hinge-region with PI3K in the same pathway. Examples of new drug discoveries that have been shown to be selective, are represented by AZD-8055, OSI-027, and INK128, which already entered clinical trials, or WYE-132, Torin1 and more are currently in preclinical studies. There are also dual PI3K/TOR inhibitors, including PI-103, GNE477, WJD008, and GSK2126458, which are reported to be promising based on their biological and clinical data [65].

JNK inhibitors have been clinically evaluated for inflammatory conditions without any unexpected adverse effects in patients. One example of a newly developed drug from cancer research is MPT0G066, which induces JNK activation, influencing cell cycle regulatory and $\mathrm{Bcl}-2$ family proteins, which triggers intrinsic apoptotic pathways. Adriamycin resulted in a timeand dose-dependent activation of JNK and treatment with vinblastine, or etoposide (VP-16) also activated JNK [66-68].

To summarize, we hypothesize, that downregulation or blockage of immune-neuronal network communication would be a target for treatment or prevention of various illnesses, including neurological and mental health disorders [101]. 
To establish the risk of developing an aberrant immune-neuronal system/communication-related disorder, one should identify its biomarkers in human saliva. Saliva is the least invasive way to collect informative data from a human at any age and has recently been shown to be a source for biomarker discoveries [102]. The development of novel screening and analyzing technology will lead to cost-effectiveness in the future, enabling us to use these individualized preventive screenings as broadly applied tools.

1. McMenamin, M., The Garden of Ediacara. Vol. 1. 1986. 178.

2. Dennett, D., Review of Other Minds: the octopus, the sea and the deep origins of consciousness: Peter Godfrey-Smith, Farrar, Straus and Giroux, NY, 2016. Vol. 34. 2019.

3. Yamamori, T. and A. Sarai, Coevolution of cytokine receptor families in the immune and nervous systems. Neurosci Res, 1992. 15(3): p. 151-61.

4. Kioussis, D. and V. Pachnis, Immune and Nervous Systems: More Than Just a Superficial Similarity? Immunity, 2009. 31(5): p. 705-710.

5. Filiano, A.J., et al., Unexpected role of interferon-gamma in regulating neuronal connectivity and social behaviour. Nature, 2016. 535(7612): p. 425-9.

6. Andersson, U. and K.J. Tracey, Neural reflexes in inflammation and immunity. J Exp Med, 2012. 209(6): p. 1057-68.

7. Arreola, R., et al., Immunomodulatory effects mediated by serotonin. J Immunol Res, 2015. 2015: p. 354957.

8. Rankin, L.C. and D. Artis, Beyond Host Defense: Emerging Functions of the Immune System in Regulating Complex Tissue Physiology. Cell, 2018. 173(3): p. 554-567.

9. Holt, P.G., Review Environmental factors and primary T-cell sensitisation to inhalant allergens in infancy: reappraisal of the role of infections and air pollution. Pediatric Allergy and Immunology, 1995. 6(1): p. 1-10.

10. Weisskopf, M.G., M.-A. Kioumourtzoglou, and A.L. Roberts, Air Pollution and Autism Spectrum Disorders: Causal or Confounded? Current environmental health reports, 2015. 2(4): p. 430-439.

11. Kim, D., et al., Psychological factors and DNA methylation of genes related to immune/inflammatory system markers: the VA Normative Aging Study. BMJ Open, 2016. 6(1): p. e009790.

12. Bendriss-Vermare, N., et al., Virus overrides the propensity of human CD4OL-activated plasmacytoid dendritic cells to produce Th2 mediators through synergistic induction of IFN-\{gamma\} and Th1 chemokine production. J Leukoc Biol, 2005. 78(4): p. 954-66.

13. Brunkhorst, A., et al., A specific role for the TFIID subunit TAF4 and RanBPM in neural progenitor differentiation. Mol Cell Neurosci, 2005. 29(2): p. 250-8.

14. Cheng, L., S. Lemmon, and V. Lemmon, RanBPM is an L1-interacting protein that regulates L1-mediated mitogen-activated protein kinase activation. Journal of neurochemistry, 2005. 94(4): p. 1102-1110.

15. Murrin, L.C. and J.N. Talbot, RanBPM, a scaffolding protein in the immune and nervous systems. J Neuroimmune Pharmacol, 2007. 2(3): p. 290-5. 
16. Camacho-Arroyo, I., L. Lopez-Griego, and J. Morales-Montor, The role of cytokines in the regulation of neurotransmission. Neuroimmunomodulation, 2009. 16(1): p. 1-12.

17. Levite, M., Neurotransmitters activate T-cells and elicit crucial functions via neurotransmitter receptors. Current opinion in pharmacology, 2008. 8(4): p. 460-471.

18. Ransohoff, R.M., Chemokines and chemokine receptors: standing at the crossroads of immunobiology and neurobiology. Immunity, 2009. 31(5): p. 711-21.

19. Farina, C., F. Aloisi, and E. Meinl, Astrocytes are active players in cerebral innate immunity. Trends Immunol, 2007. 28(3): p. 138-45.

20. Kielian, T., Toll-like receptors in central nervous system glial inflammation and homeostasis. J Neurosci Res, 2006. 83(5): p. 711-30.

21. Prehaud, C., et al., Virus infection switches TLR-3-positive human neurons to become strong producers of beta interferon. J Virol, 2005. 79(20): p. 12893-904.

22. Rose-John, S., et al., The IL-6/sIL-6R complex as a novel target for therapeutic approaches. Expert Opin Ther Targets, 2007. 11(5): p. 613-24.

23. Lee, S.C., et al., Cytokine production by human fetal microglia and astrocytes. Differential induction by lipopolysaccharide and IL-1 beta. J Immunol, 1993. 150(7): p. 2659-67.

24. Sebire, G., et al., In vitro production of $I L-6, I L-1$ beta, and tumor necrosis factor-alpha by human embryonic microglial and neural cells. J Immunol, 1993. 150(4): p. 1517-23.

25. Hariri, R.J., et al., Traumatic injury induces interleukin-6 production by human astrocytes. Brain Res, 1994. 636(1): p. 139-42.

26. Van Wagoner, N.J., et al., Interleukin-6 (IL-6) production by astrocytes: autocrine regulation by IL-6 and the soluble IL-6 receptor. J Neurosci, 1999. 19(13): p. 5236-44.

27. Ma, X., et al., IL-17 enhancement of the IL-6 signaling cascade in astrocytes. J Immunol, 2010. 184(9): p. 4898-906.

28. Turka, L.A., et al., Interleukin 12: a potential link between nerve cells and the immune response in inflammatory disorders. Molecular medicine (Cambridge, Mass.), 1995. 1(6): p. 690-699.

29. Watkins, L.R., et al., Blockade of interleukin-1 induced hyperthermia by subdiaphragmatic vagotomy: evidence for vagal mediation of immune-brain communication. Neurosci Lett, 1995. 183(1-2): p. 27-31.

30. Watkins, L.R., S.F. Maier, and L.E. Goehler, Cytokine-to-brain communication: a review \& analysis of alternative mechanisms. Life Sci, 1995. 57(11): p. 1011-26.

31. Nathan, C. and A. Ding, Nonresolving inflammation. Cell, 2010. 140(6): p. 871-82.

32. Tracey, K.J., Physiology and immunology of the cholinergic antiinflammatory pathway. The Journal of clinical investigation, 2007. 117(2): p. 289-296.

33. Meves, H., The action of prostaglandins on ion channels. Current neuropharmacology, 2006. 4(1): p. 41-57.

34. Parnell, E., T.M. Palmer, and S.J. Yarwood, The future of EPAC-targeted therapies: agonism versus antagonism. Trends in pharmacological sciences, 2015. 36(4): p. 203-214.

35. Wheway, J., H. Herzog, and F. Mackay, NPY and receptors in immune and inflammatory diseases. Curr Top Med Chem, 2007. 7(17): p. 1743-52.

36. Wolf, S.A., S. Tauber, and O. Ullrich, CNS immune surveillance and neuroinflammation: endocannabinoids keep control. Curr Pharm Des, 2008. 14(23): p. 2266-78.

37. Khan, A.A., et al., Physiological regulation of the immunological synapse by agrin. Science, 2001. 292(5522): p. 1681-6. 
38. Sloan, R.P., et al., $R R$ interval variability is inversely related to inflammatory markers: the CARDIA study. Molecular medicine (Cambridge, Mass.), 2007. 13(3-4): p. 178-184.

39. Wallrapp, A., et al., The neuropeptide NMU amplifies ILC2-driven allergic lung inflammation. Vol. 549. 2017.

40. Allen, N.D., Temporal and epigenetic regulation of neurodevelopmental plasticity. Philosophical transactions of the Royal Society of London. Series B, Biological sciences, 2008. 363(1489): p. 23-38.

41. Jessell, T.M., Neuronal specification in the spinal cord: inductive signals and transcriptional codes. Nat Rev Genet, 2000. 1(1): p. 20-9.

42. Foster, K., et al., Contribution of neural crest-derived cells in the embryonic and adult thymus. J Immunol, 2008. 180(5): p. 3183-9.

43. Creuzet, S., G. Couly, and N.M. Le Douarin, Patterning the neural crest derivatives during development of the vertebrate head: insights from avian studies. Journal of anatomy, 2005. 207(5): p. 447-459.

44. Wilson, C.B., E. Rowell, and M. Sekimata, Epigenetic control of T-helper-cell differentiation. Nat Rev Immunol, 2009. 9(2): p. 91-105.

45. Landis, S.C., Target regulation of neurotransmitter phenotype. Trends in Neurosciences, 1990. 13(8): p. 344-350.

46. Zhou, L., M.M. Chong, and D.R. Littman, Plasticity of CD4+ $T$ cell lineage differentiation. Immunity, 2009. 30(5): p. 646-55.

47. Veiga-Fernandes, H., et al., Tyrosine kinase receptor RET is a key regulator of Peyer's patch organogenesis. Nature, 2007. 446(7135): p. 547-51.

48. Patel, A., et al., Differential RET Signaling Pathways Drive Development of the Enteric Lymphoid and Nervous Systems. Science Signaling, 2012. 5(235): p. ra55.

49. Mizui, M., A. Kumanogoh, and H. Kikutani, Immune semaphorins: novel features of neural guidance molecules. J Clin Immunol, 2009. 29(1): p. 1-11.

50. Bajenoff, M., et al., Stromal cell networks regulate lymphocyte entry, migration, and territoriality in lymph nodes. Immunity, 2006. 25(6): p. 989-1001.

51. Agoston, D.V., et al., Ikaros is expressed in developing striatal neurons and involved in enkephalinergic differentiation. J Neurochem, 2007. 102(6): p. 1805-1816.

52. McMillan, B.J. and C.A. Bradfield, The aryl hydrocarbon receptor sans xenobiotics: endogenous function in genetic model systems. Mol Pharmacol, 2007. 72(3): p. 487-98.

53. Veitenhansl, M., et al., 40(th) EASD Annual Meeting of the European Association for the Study of Diabetes : Munich, Germany, 5-9 September 2004. Diabetologia, 2004. 47(Suppl 1): p. A1-A464.

54. Chen, G.-L. and G.M. Miller, Tryptophan hydroxylase-2: An emerging therapeutic target for stress disorders. Biochemical Pharmacology, 2013. 85(9): p. 1227-1233.

55. Leonard, B.E., The concept of depression as a dysfunction of the immune system. Current immunology reviews, 2010. 6(3): p. 205-212.

56. Rossignol, D.A. and R.E. Frye, Melatonin in autism spectrum disorders. Curr Clin Pharmacol, 2014. 9(4): p. 326-34.

57. Kaneko, I., et al., 1,25-Dihydroxyvitamin D regulates expression of the tryptophan hydroxylase 2 and leptin genes: implication for behavioral influences of vitamin D. The FASEB Journal, 2015. 29(9): p. 4023-4035. 
58. Zhang, Y., et al., Methylation of the tryptophan hydroxylase2 gene is associated with mRNA expression in patients with major depression with suicide attempts. Mol Med Rep, 2015. 12(2): p. 3184-90.

59. Jarome, T.J., et al., EZH2 Methyltransferase Activity Controls Pten Expression and mTOR Signaling during Fear Memory Reconsolidation. J Neurosci, 2018. 38(35): p. 7635-7648.

60. Mihaylova, M.M. and R.J. Shaw, The AMPK signalling pathway coordinates cell growth, autophagy and metabolism. Nat Cell Biol, 2011. 13(9): p. 1016-23.

61. Chakrabarti, S., et al., Infection-induced host translational blockage inhibits immune responses and epithelial renewal in the Drosophila gut. Cell Host Microbe, 2012. 12(1): p. 60-70.

62. Zhu, Y., et al., Rap2-JNK Removes Synaptic AMPA Receptors during Depotentiation. Neuron, 2005. 46(6): p. 905-916.

63. Yarza, R., et al., c-Jun N-terminal Kinase (JNK) Signaling as a Therapeutic Target for Alzheimer's Disease. Frontiers in pharmacology, 2016. 6: p. 321-321.

64. Basu, S., et al., Suppression of MAPK/JNK-MTORC1 signaling leads to premature loss of organelles and nuclei by autophagy during terminal differentiation of lens fiber cells. Autophagy, 2014. 10(7): p. 1193-1211.

65. Schenone, S., et al., ATP-competitive inhibitors of mTOR: an update. Curr Med Chem, 2011. 18(20): p. 2995-3014.

66. Osborn, M.T. and T.C. Chambers, Role of the stress-activated/c-Jun NH2-terminal protein kinase pathway in the cellular response to adriamycin and other chemotherapeutic drugs. J Biol Chem, 1996. 271(48): p. 30950-5.

67. Huang, H.-L., et al., MPTOG066, a novel anti-mitotic drug, induces JNK-independent mitotic arrest, JNK-mediated apoptosis, and potentiates antineoplastic effect of cisplatin in ovarian cancer. Scientific Reports, 2016. 6: p. 31664.

68. Ferrao, P.T., Taking out the JNK: A window of opportunity to improve cancer therapy. Molecular \& cellular oncology, 2016. 3(3): p. e1128515-e1128515.

69. Sara, S.J., The locus coeruleus and noradrenergic modulation of cognition. Nat Rev Neurosci, 2009. 10(3): p. 211-23.

70. Berridge, C.W. and B.D. Waterhouse, The locus coeruleus-noradrenergic system: modulation of behavioral state and state-dependent cognitive processes. Brain Res Brain Res Rev, 2003. 42(1): p. 33-84.

71. Katsuki, H., Y. Izumi, and C.F. Zorumski, Noradrenergic regulation of synaptic plasticity in the hippocampal CA1 region. J Neurophysiol, 1997. 77(6): p. 3013-20.

72. O'Dell, T.J., et al., beta-Adrenergic receptor signaling and modulation of long-term potentiation in the mammalian hippocampus. Learn Mem, 2015. 22(9): p. 461-71.

73. $\mathrm{Hu}, \mathrm{H}$., et al., Emotion Enhances Learning via Norepinephrine Regulation of AMPA-Receptor Trafficking. Cell, 2007. 131(1): p. 160-173.

74. Tully, K. and V.Y. Bolshakov, Emotional enhancement of memory: how norepinephrine enables synaptic plasticity. Molecular Brain, 2010. 3(1): p. 15.

75. Lazzaro, S.C., et al., Antagonism of lateral amygdala alpha1-adrenergic receptors facilitates fear conditioning and long-term potentiation. Learning \& Memory, 2010. 17(10): p. 489-493.

76. Esler, M., et al., The neuronal noradrenaline transporter, anxiety and cardiovascular disease. J Psychopharmacol, 2006. 20(4 Suppl): p. 60-6. 
77. Johnson, L., et al., Regulation of the Fear Network by Mediators of Stress: Norepinephrine Alters the Balance between Cortical and Subcortical Afferent Excitation of the Lateral Amygdala. Frontiers in Behavioral Neuroscience, 2011. 5(23).

78. Charney, D.S., Neuroanatomical circuits modulating fear and anxiety behaviors. Acta Psychiatr Scand Suppl, 2003(417): p. 38-50.

79. McGaugh, J.L., The amygdala modulates the consolidation of memories of emotionally arousing experiences. Annu Rev Neurosci, 2004. 27: p. 1-28.

80. Klann, E., et al., Synaptic plasticity and translation initiation. Learn Mem, 2004. 11(4): p. 365-72.

81. Gelinas, J.N., et al., ERK and mTOR signaling couple beta-adrenergic receptors to translation initiation machinery to gate induction of protein synthesis-dependent long-term potentiation. J Biol Chem, 2007. 282(37): p. 27527-35.

82. Takei, N. and H. Nawa, mTOR signaling and its roles in normal and abnormal brain development. Frontiers in Molecular Neuroscience, 2014. 7(28).

83. Hoeffer, C.A. and E. Klann, mTOR signaling: at the crossroads of plasticity, memory and disease. Trends in neurosciences, 2010. 33(2): p. 67-75.

84. Graber, T.E., P.K. McCamphill, and W.S. Sossin, A recollection of $m$ TOR signaling in learning and memory. Learn Mem, 2013. 20(10): p. 518-30.

85. Mac Callum, P.E., et al., Systemic inhibition of mTOR kinase via rapamycin disrupts consolidation and reconsolidation of auditory fear memory. Neurobiol Learn Mem, 2014. 112: p. 176-85.

86. Bliss, T.V. and A.R. Gardner-Medwin, Long-lasting potentiation of synaptic transmission in the dentate area of the unanaestetized rabbit following stimulation of the perforant path. J Physiol, 1973. 232(2): p. 357-74.

87. Bliss, T.V. and G.L. Collingridge, A synaptic model of memory: long-term potentiation in the hippocampus. Nature, 1993. 361(6407): p. 31-9.

88. Bear, M.F. and R.C. Malenka, Synaptic plasticity: LTP and LTD. Curr Opin Neurobiol, 1994. 4(3): p. 389-99.

89. Kandel, E.R., The molecular biology of memory storage: a dialog between genes and synapses. Biosci Rep, 2001. 21(5): p. 565-611.

90. Maity, S., et al., Noradrenaline goes nuclear: epigenetic modifications during long-lasting synaptic potentiation triggered by activation of beta-adrenergic receptors. J Physiol, 2016. 594(4): p. 863-81.

91. Nathan, C., Points of control in inflammation. Nature, 2002. 420(6917): p. 846-52.

92. Besedovsky, H.O. and A. del Rey, Immune-neuro-endocrine interactions: facts and hypotheses. Endocr Rev, 1996. 17(1): p. 64-102.

93. Mosser, D.M. and X. Zhang, Interleukin-10: new perspectives on an old cytokine. Immunol Rev, 2008. 226: p. 205-18.

94. Wong, C.H.Y., et al., Functional Innervation of Hepatic iNKT Cells Is Immunosuppressive Following Stroke. Science, 2011. 334(6052): p. 101.

95. Traynor, B.J., et al., Clinical features of amyotrophic lateral sclerosis according to the El Escorial and Airlie House diagnostic criteria: A population-based study. Arch Neurol, 2000. 57(8): p. 1171-6.

96. Ingre, C., et al., Risk factors for amyotrophic lateral sclerosis. Clinical epidemiology, 2015. 7: p. 181-193.

97. Xue, Y.C., et al., Enteroviral Infection: The Forgotten Link to Amyotrophic Lateral Sclerosis? Frontiers in Molecular Neuroscience, 2018. 11(63). 
98. Breit, S., et al., Vagus Nerve as Modulator of the Brain-Gut Axis in Psychiatric and Inflammatory Disorders. Front Psychiatry, 2018. 9: p. 44.

99. Niijima, A., Reflex Effects of Oral, Gastrointestinal and Hepatoportal Glutamate Sensors on Vagal Nerve Activity. The Journal of Nutrition, 2000. 130(4): p. 971S-973S.

100. Tracey, K.J., The inflammatory reflex. Nature, 2002. 420(6917): p. 853-9.

101. Evans, E.A., T. Kawli, and M.-W. Tan, Pseudomonas aeruginosa Suppresses Host Immunity by Activating the DAF-2 Insulin-Like Signaling Pathway in Caenorhabditis elegans. PLOS Pathogens, 2008. 4(10): p. e1000175.

102. Yoshizawa, J.M., et al., Salivary biomarkers: toward future clinical and diagnostic utilities. Clinical microbiology reviews, 2013. 26(4): p. 781-791. 